\title{
Quantitative evaluation of mechanical properties in tissue- engineered auricular cartilage
}

\section{Review Article}

Author(s):

Nimeskern, Luc; van Osch, Gerjo J.V.M.; Müller, Ralph (D); Stok, Kathryn S.

Publication date:

2014-02

Permanent link:

https://doi.org/10.3929/ethz-a-010577328

Rights / license:

In Copyright - Non-Commercial Use Permitted

Originally published in:

Tissue Engineering. Part B, Reviews 20(1), https://doi.org/10.1089/ten.teb.2013.0117 
Quantitative evaluation of mechanical properties in tissue-engineered auricular cartilage

Luc Nimeskern ${ }^{1}$, Gerjo J. V. M. van Osch ${ }^{2}$, Ralph Müller ${ }^{1}$, and Kathryn S. Stok ${ }^{1}$

1: Institute for Biomechanics, ETH Zurich, Zurich, Switzerland

2: Department of Otorhinolaryngology and Department of Orthopaedics, Erasmus MC, University Medical Center Rotterdam, Rotterdam, The Netherlands.

\section{Funding sources:}

This study was supported by the Swiss National Science Foundation (NRP63) and ERA-NET/EuroNanoMed (EAREG-406340-131009/1).

\section{Running title:}

Mechanical evaluation of tissue-engineered auricular cartilage

\section{Authors:}

Luc Nimeskern
tel: $\quad+41446333104$
fax: $\quad$ +414463315 73
email: nluc@ethz.ch

Gerjo J. V. M. van Osch
tel: $\quad$ +31 107043661
fax: $\quad+31107044690$
email: g.vanosch@erasmusmc.nl

Ralph Müller
tel: $\quad+41446324592$
fax: $\quad+41446321214$
email: ram@ethz.ch

Kathryn S. Stok 
15.02 .13
tel: $\quad$ +4144632 4580
fax: $\quad$ +41446331573
email: kas@ethz.ch

\section{Corresponding author:}

Kathryn S. Stok, PhD.

Institute for Biomechanics, ETH Zürich

Wolfgang-Pauli-Strasse 10

8093 Zürich

Switzerland

Tel: +41446324580

Fax: +41 446331573

Email: kas@ethz.ch

\section{Number of words/characters:}

abstract $=246$

manuscript (not including references) $=5054$

\section{Number Figures:}

color $=1$

$b / w=5$

\section{Keywords:}

Auricular cartilage, elastic cartilage, auricle, pinna, outer ear, mechanical testing, elastin

\section{Conflict of interest:}

All authors have no conflict of interest. 


\section{Abstract}

Tissue-engineering (TE) efforts for ear-reconstruction often fail due to mechanical incompetency. It is therefore key for successful auricular cartilage TE to ensure functional competency, i.e. to mimic the mechanical properties of the native ear tissue. A review of past attempts to engineer auricular cartilage shows unsatisfactory functional outcomes with various cell-seeded biodegradable polymeric scaffolds in immunocompetent animal models. However, promising improvements to construct stability were reported with either mechanically-reinforced scaffolds or novel two-stage implantation techniques. Nonetheless, quantitative mechanical evaluation of the constructs is usually overlooked, and such an evaluation of TE constructs alongside a benchmark of native auricular cartilage would allow real-time monitoring and improve functional outcomes of auricular TE strategies.

Although quantitative mechanical evaluation techniques are readily available for cartilage, these techniques are designed to characterize the main functional components of hyaline and fibrous cartilage such as the collagen matrix or the glycosaminoglycan (GAG) network, but they overlook the functional role of elastin, which is a major constituent of auricular cartilage. Hence for monitoring auricular cartilage TE, novel evaluation techniques need to be designed. These should include a characterization of the specific composition and architecture of auricular cartilage, as well as mechanical evaluation of all functional components.

Therefore, this paper reviews the existing literature on auricular cartilage TE as well as cartilage mechanical evaluation and proposes recommendations for designing a mechanical evaluation protocol specific for auricular cartilage, and establishing a benchmark for native auricular cartilage to be used for quantitative evaluation of TE auricular cartilage. 


\section{Introduction}

Microtia and trauma are the two main causes for permanent alteration of the shape of the auricle - the external part of the ear. Microtia is a congenital anomaly with severity ranking from auricles smaller than expected to severe attenuation of the entire auricle. Multinational registries of congenital malformations suggest the prevalence of microtia ranges from 0.76 to 2.35 per 10000 (1) with most cases being monolateral (2). Additionally, since the auricle is composed mainly of cartilage, an avascular and aneural tissue which does not regenerate once damaged, any trauma, (burns, dog bites, tissue resection or X-ray therapy for cancer treatment) leads to permanent deformities.

Auricular cartilage $(A \cup C)$ is present in all parts of the auricle except the lobule which is composed of adipose tissue (1). It is anchored on the human head through a largely vestigial musculature, see figure 1 and surrounded by a perichondrium that connects it to the highly vascularized skin which provides the necessary nutrients (1).

AUC is classified as elastic cartilage and differs in its structure and function from the other two cartilage subtypes: hyaline and fibrocartilage. The distinction between the three cartilage subtypes is based on the presence of collagen and elastic fibers, as well as tissue morphology (3). Various cartilaginous tissues are classified as hyaline cartilage in the human body, e.g. articular, nasoseptal, or costal cartilage. Fibrocartilage is found in the intervertebral disks and in the menisci, and also connects tendons to bones. Elastic cartilage is present in the auricle, the epiglottis and at the tip of the nose (4), see figure 2 . Whereas hyaline and fibrocartilage have been extensively characterized (5-7) little data is available on the mechanical properties of AUC (8).

Surgical reconstruction with autologous cartilage is currently the only existing treatment for AUC defects. Tissue-engineering (TE) of AUC is a potential alternative that may advantageously reduce the donor-site morbidity resulting from surgical reconstruction (9). A crucial role is played by the mechanical properties of the engineered cartilage that should be both consistent with those of native cartilage and also allow surgical handling during implantation. For example, if TE AUC is too soft, it would not be able to maintain its shape or to withstand the many loads placed on it during daily life (e.g. sleeping, wearing earphones, helmets etc.). Conversely, if the auricle is too stiff, it would cause discomfort to the patient, and also undergo extrusion as observed with regular alloplastic implants (10). 
Most publications in the field of auricular tissue engineering however overlook mechanical testing. Additionally, very little is known about the mechanical properties of AUC, and thus an appropriate benchmark for comparison is unavailable. For these reasons, the auricular TE field would benefit from a precise characterization of the mechanical properties desired for AUC grafts. This benchmark would then allow for assessment and monitoring of engineered cartilage constructs during cultivation, to decide whether they match the necessary functional competence.

Therefore this article firstly reviews the past attempts to reconstruct and engineer AUC as well as their functional outcomes. Secondly, the existing mechanical evaluation methods for cartilage, with an emphasis on the specific structure of $A U C$ and the consequences for its mechanical properties are reviewed. Finally recommendations for future research aimed at achieving the desired mechanical properties through adequate mechanical evaluation are discussed.

\section{Functional outcome of auricular cartilage reconstruction and engineering techniques}

\section{a. Surgical reconstruction: state-of-the-art treatment of auricular defects}

Surgical reconstruction with autologous cartilage is currently the only existing treatment for auricular defects. Surgical ear reconstruction was developed by Tanzer in the late 1950s (11), and today, the most frequently applied methods are based on the work of Brent (12) and Nagata (13). These procedures require several consecutive surgeries with the complexity of the operation varying with the size of the defect. For total reconstruction, firstly autologous cartilage is harvested from the ribs or the nasal septum, then an auricle framework is fabricated with the harvested material, see figure 3. Implantation is then performed in 4 stages (12) or 2 stages (13). The autologous framework is first implanted subcutaneously (step 1), i.e. there is no separation between the grafted auricle and the skull. In steps 2 and 3, the remnants of AUC are used to complete the implanted framework and the tragus is reconstructed. In step 4 , the reconstructed auricle is separated from the head after skin expansion. Two-stage implantation techniques combine the first three steps into a single surgery (14). The main advantage of the 2-stage techniques is that they are less time consuming. However, they are more challenging, thus requiring experienced surgeons (14). Alternatively, alloplastic implants such as high density porous polyethylene (HDPE) frameworks can be used instead of autologous tissue to construct the auricle (15). Due to risks of complication related to staged and time-consuming surgery, surgical reconstruction is not recommended in patients with high comorbidity, e.g. aged patients or patient 
with poor local tissues resulting from radiation, cancer, or prior surgery. In such cases, prostheses anchored with clips or magnets on osseointegrated implants are preferred (16).

The main drawback of surgical reconstruction is the donor-site morbidity following cartilage harvesting. The sixth, seventh, and eighth costal ribs are usually harvested for cartilage. The sixth and seventh ribs are used to form the auricular body framework, whereas the "floating" eighth rib is used to fabricate the helical element (16). The large quantity of cartilage harvested causes acute post-operative pain and may lead to lung collapse or thorax deformities especially in young patients (17). Engineering cartilage instead of harvesting would advantageously reduce this donor-site morbidity.

\section{b. Auricular cartilage tissue-engineering}

Over the past decade multiple groups have investigated the possibility of engineering cartilage in the shape of a 3D auricle. As in any TE application, scaffolds are used to create an adequate mechanical and biochemical environment, which should promote cell adhesion and proliferation. Additionally, in the case of auricular TE, the scaffold is shaped into a $3 \mathrm{D}$ auricle-like shape that should be retained until the cartilage itself is able to maintain the shape of the construct.

The most investigated scaffold materials are biodegradable polymers, for instance poly(lactic acid) (PLA, PLLA) (18-20), poly(glycolic acid) (PGA) (18-22), poly- $\varepsilon$ caprolactone (PCL) (20), polyhydroxybutyrate (PHB) (20), and their copolymers (23-27). These different polymer combinations allow tuning of the mechanical properties as well as the degradation rate of the scaffold. The longest in vivo studies reported were performed over a period of 40 weeks $(20,25,26)$. Among these, those performed in immuno-compromised mouse models $(20,25,26)$ reported cartilage formation and adequate shape stability at 40 weeks, where shape stability was assessed qualitatively (visual evaluation of shape and size). Shieh et al. (20) were the only ones to perform their work in an immuno-competent animal model, and observed a severe foreign body reaction after 3 months in all investigated polymeric scaffolds (PCL, PHB and PGA coated with PLLA) leading to collapse of the TE constructs. The foreign body reaction was attributed to the degradation products of the scaffold. The loss of shape stability was presumably due to the resulting inflammation as well as the observed higher skin contraction.

In order to avoid foreign body reactions with biodegradable polymers, alternative approaches have been investigated. Saim et al. (28) injected a hydrogel scaffold (Pluronic ${ }^{\circledR}$ F127, a mixture of polyethylene oxide and polypropylene oxide) seeded with autologous chondrocytes in an auricular-shaped skin-fold channel of immuno-competent Yorkshire swine. No immune reaction was detected. Ten weeks after implantation they 
observed the formation of a cartilage extracellular matrix (ECM) and histological results consistent with AUC. The volume of the construct remained constant, qualitative evaluation showed that flexibility was consistent with AUC, yet no state-of-the-art quantitative mechanical evaluation was performed. Also one cannot conclude from the results that it would be able to maintain a 3D shape. In order to create 3D constructs, Xu et al. (29) incorporated two layers of lyophilized swine perichondrium to a chondrocyte-seeded fibrin hydrogel. The constructs were additionally supported by external stenting for the first 6 weeks of in vivo implantation (immuno-compromised model). After 12 weeks of in vivo implantation cartilage formation was reported, as well as satisfying qualitative evaluation of size, shape and flexibility, yet again no quantitative mechanical evaluation was performed. Injection molding of chondrocytes in fibrin glue (30) or calcium alginate (31) was also proposed, and while fibrin glue constructs demonstrated poor shape stability and weak mechanical properties (apparent modulus) four weeks after implantation in nude mice (30), calcium alginate constructs showed equilibrium modulus values equivalent to native AUC 30 weeks after implantation in an immunocompetent sheep model (31). No ear-shaped constructs have been reported with this technique. The methods of Kamil et al. (22) and Neumeister et al. (32), describe non-biodegradable molds (a gold or a silicon mold, respectively) filled with a chondrocyte-seeded hydrogel implanted in vivo, which are used to generate AUC in the desired shape and with adequate mechanical properties, which could then be reimplanted into a patient to treat a specific defect. Both studies observed the formation of auricle-shaped cartilage, yet no tests were performed to assess maintenance of cartilage shape after reimplantation or mechanical properties.

As seen previously, hydrogel-based constructs were used in most studies in combination with either nonbiodegradable molds or external stenting, because the constructs alone could not maintain the 3D shape. In order to combine advantageous hydrogel properties with mechanical stability, several groups have investigated composite implants with internal non-biodegradable core surrounded by cell-seeded scaffold materials such as collagen (33), fibrin gels $(34,35)$ or alginate (36). Zhou et al. (33) proposed a chondrocyte-seeded collagen scaffold reinforced by a permanent titanium framework. Cartilage ECM and weak elastin production was reported after 2 weeks of in vitro cultivation followed by 6 weeks of implantation in nude mice. No extrusion of the titanium wire was observed. Construct flexibility was assessed, but no quantitative assessment of mechanical competence was performed. Lee et al. (34) described the use of HDPE implants covered with chondrocyte-fibrin constructs. After 24 weeks implantation in nude mice mature cartilage formation was reported, yet no mechanical evaluation was performed on the implants. Similarly, Bichara et al. report the 
injection of an alginate-chondrocyte mix in a porous poly(vinyl alcohol), PVA, implant. Six weeks after implantation in nude mice, neocartilage formation was reported with promising increases in mechanical strength (36). However, the suitability of the test for cartilage function is disputed and the comparison to native tissue is missing. Currently, composite constructs are favored by researchers, and despite results showing no extrusion of non-biodegradable components after up to 24 weeks of implantation in immunocompromised animal models, further investigation is required to assess the extrusion risk in the human body over the patient lifetime, as well as mechanical comparison with native AUC.

A promising alternative to the use of composite constructs has been proposed by Yanaga et al. (37). Thanks to a scaffold-free two-step technique, they reported successful AUC TE in four human patients over several years. In a first step, a multilayered chondrocyte culture was implanted in a non-load bearing region (lower abdomen) for 6 months. In a later step, the constructs were harvested, carved into the shape of an auricle and implanted at the location of the auricular defect. Histological examination showed the formation of elastic cartilage during maturation in the lower abdomen. No quantitative mechanical evaluation was performed, nonetheless during 2-5 years postoperative monitoring, shape maintenance without absorption of the neo-cartilage was reported. As this technique does not require any scaffold material, it is free of scaffold related issues. Its main drawback is again the need for two successive surgeries. The key aspect of this approach is the first implantation in a highly vascularized, non-load bearing area where a mature cartilage block with perichondrium-like vascular supply is grown.

Beside mechanical competence, another challenge of AUC TE is to obtain a sufficient number of chondrogenic cells to seed a human-size ear scaffold (38). Auricular chondrocytes isolated from patient biopsies require in vitro monolayer expansion due to the small cell yield $(23,27,32,34,36,37)$, however this is known to lead to de-differentiation $(39,40)$, i.e the cells cannot synthesize cartilage matrix (41). 3D culture systems with adequate seeding density (42) or growth factors (43-46) have shown promise in the re-dedifferentiation of expanded chondrocyte cultures, or alternatively, adipose tissue or bone marrow-derived mesenchymal stem cells can be harvested from the patient, expanded and differentiated to a chondrogenic lineage $(47,48)$. No ear-shaped scaffolds with stem-cell derived chondrocytes have been reported in literature, nor is it known how the cell source will influence the functional properties of the construct.

In light of these results, it appears that mature elastic cartilage ECM with functional mechanical properties at the time of implantation, are critical to guarantee the success of a TE auricle. Functional evaluation has often 
been overlooked, where most authors $(18-23,25-29,32,35,49,50)$ report only qualitative evaluation of size, shape maintenance or flexibility. A limited number of publications report quantitative mechanical evaluation, yet with diverse testing protocols $(24,30,31,36,51)$ and often without comparison to native AUC $(24,30,36)$. Therefore, it can be reasoned that the auricular TE field would benefit from a precise characterization of the mechanical properties of native AUC, in order to set a benchmark against which to assess the functional competence of engineered AUC constructs. The second part of this review concentrates on the mechanical characterization methods available for native and TE cartilage; with a focus on their relevance to the evaluation of AUC.

\section{Mechanical evaluation of auricular cartilage}

Cartilage composition and structure is known to be closely related to its mechanical properties (52). It is therefore necessary to understand the functional role of tissue components in order to identify adequate testing methods.

\section{a. Tissue composition}

The main components of AUC are water, glycosaminoglycan, collagen fibers and elastin fibers (8); see figure 4 (a). Histology sections stained for the main components of the matrix are displayed in figure 5. Elastin is extensively present in AUC and is considered to be the major difference between AUC and the other cartilage subtypes (8). Previous publications report little elastin in hyaline and fibrocartilage $(8,53)$. The potential functional role of elastin in intervertebral disc $(54,55)$ and articular cartilage $(56-58)$ has only recently been investigated.

Elastin is a hydrophobic array of cross-linked tropoelastin proteins (59), which is a durable biopolymer with a low turnover in healthy tissue (60). Elastin fibers include three different types of fibers with different structures: oxytalan fibers, elaunin fibers, and elastic fibers (61), see figure 6 . In the literature, these three fibers are often indiscriminately referred to as 'elastic fibers', 'elastin', or more precisely 'elastin-related fibers'. Oxytalan fibers are devoid of elastin (61) and consist of bundles of microfibrils (10 - $14 \mathrm{~nm}$ in diameter), which themselves are made up of at least five distinct proteins (62) including fibrillin (63). Elaunin fibers are composed of microfibrils and cross-linked elastin proteins (61). While microfibrils are the major component of elaunin fibers, elastin predominates in elastic fibers (61) and makes up the bulk of the fiber (64), see figure 4 (c). Cotta-Pereira et al. (65) proposed that the three different elastin-related fibers may represent interruptions in successive phases of the maturation of elastic fibers, e.g. a bundle of microfibrils resembling an oxytalan 
fiber could be either an immature elastic fiber or a mature oxytalan fiber. While the elastin protein displays the ability to recoil after being stretched (60) and therefore provides resilience to the fiber, microfibrils are suggested to have an anchoring function at various anatomical locations, e.g. linking the lens to the ciliary body in the eye, connecting bone and tooth cementum in the periodontal ligament, and binding epidermis to the underlying dermis in skin (61). Given the different relative amount of elastin and microfibrils between elastic, elaunin and oxytalan fibers it has been suggested that these fibers have different mechanical properties (61). Molecular combining experiments have shown that fibrillin-rich microfibrils are two orders of magnitude stiffer than elastin (66). Elaunin fibers, which contain less elastin than elastic fibers, are therefore expected to display elastic properties intermediate to those of elastic fibers and oxytalan fibers. Till now there are no reports in literature of the relative content of these fibers in mature AUC.

While elastin is almost exclusively found in AUC (among the cartilage subtypes), the other tissue components are common to all types of cartilage (8). Proteoglycan consists of a central protein core to which up to 150 polysaccharide chains (glycosaminoglycans) are attached, see figure 4 (b). Numerous proteoglycan molecules bind to a long chain of hyaluronate forming a macromolecule that is immobilized in the collagen network (67). The carboxyl and sulfate groups present on the glycosaminoglycan chains form negatively-charged ions which are known to play a role in cartilage mechanical properties due to charge-dependent osmotic swelling pressures (5). This osmotic swelling pressure is counterbalanced by the collagen network which in doing so develops internal tensile stresses (67), see figure 4 (d). Nauman et al. (8) reported a significantly lower GAG content in AUC (epiglottal and auricular) than in hyaline cartilage (nasal and articular) in the rabbit. Variation in collagen composition between the different cartilage subtypes has also been reported. The fibrocartilage of the intervertebral disc contains mostly collagen type-I and type-II (68). Collagen type-II is predominant in articular hyaline cartilage (more than 90\%), after type-XI ( 3\%), and type-IX ( 1\%) (69). Similarly, the collagen network of AUC is reported to be predominantly type-II $(8,70)$.

Collagen crosslinking has been shown to be important for functional cartilage matrix (71). Concerning elastin crosslinking, Elbjeirami et al. (72) have shown that increased desmosine crosslinking, via lysyl oxidase activity, enhance the mechanical properties of vascular smooth muscle cell-populated tissue-engineered constructs. As formation of the elastin-specific crosslinks desmosine is necessary for the synthesis of elastin from its soluble precursor, tropopelastin (59), it is anticipated that desmosine crosslink density influences elastin mechanical properties. 


\section{b. Mechanical testing of cartilage and tissue-engineered cartilage: state-of-the-art}

Two distinct mechanisms are involved in the response of cartilage to compressive loading: the intrinsic mechanical properties of the ECM, and the resistance to interstitial water flow through the ECM (5), which is governed by the permeability and swelling pressure (Donnan osmotic pressure) of the ECM (67). During compressive loading, there is an instantaneous response involving a change in shape of the material without change in volume (instantaneous modulus); followed by a transient phase where the fibrillar stress relaxes and fluid flows out of the matrix. Finally, the tissue reaches an equilibrium or steady-state response where fluid flow ceases; then the load is carried by the solid matrix, and is characterized by the equilibrium modulus (73). The equilibrium modulus of articular cartilage increases with increasing GAG content (52) and decreases with increasing water content (74). These experimental observations can be attributed at least partially to the mechanism proposed for the loading behavior of cartilage; i.e. a higher content of negatively charged GAG creates higher charge density in the porous matrix which results in an increased osmotic swelling pressure in the tissue, and vice versa (67). Creep and stress relaxation tests are commonly performed to characterize these properties (67) using confined or unconfined compression as well as indentation testing (75).

During confined compression creep a cartilage plug is placed in a rigid, close-fitting cylindrical chamber, and tested in uniaxial compression (74). Fluid can escape from the bottom or the top of the chamber through porous platens, and as it is forced out, the specimen "creeps", and equilibrium is reached when all fluid flow stops and the specimen no longer deforms. If the test is repeated at successively higher levels of stress, the resulting equilibrium stress-strain curve is approximately linear for strains below about $20 \%$ (67). This behavior is usually expressed in terms of equilibrium modulus or aggregate modulus, and equals the slope of the equilibrium stress-strain curve (67). Alternatively, confined compression stress-relaxation can be performed, where increasing strain steps are applied (67). Again, the equilibrium modulus is measured as the slope of the equilibrium stress-strain curve. The instantaneous modulus is also captured by stress-relaxation test and equals the slope of the stress-strain curve during the first strain application. Instantaneous modulus is dependent on the applied strain rate (67). Confined compression set ups have several limitations. Firstly, inappropriate description of the boundary conditions at the interface between the porous platens and the sample can cause the measured material properties to be different from the true ones (76). Secondly, computational modeling shows that friction between the sample and the chamber can influence the measurements (77). 
Another commonly used compression test is indentation $(78,79)$. In this test, a porous or non-porous indenter is used to indent the sample. The diameter of the indenter is chosen to allow fluid flow, while the sample is confined radially. Indentation can be performed in situ, e.g. directly on an articular joint (80), or at different locations of the same tissue-engineered sample, so as to evaluate spatial variation in mechanical properties (81). However, it has been shown that the measured properties are influenced by friction at the tissue/indenter interface, particularly for thin samples (82). Therefore indentation results from different publications should be compared with caution.

Unconfined compression tests are rarely performed on cartilage (83). Here, the cartilage is compressed between two perfectly smooth, and non-porous plates. Ideally, pure compression takes place, yet practically it is not possible to avoid shear stresses due to friction between the sample and the platens (83). To reduce this effect lubrication solution, such as synovial fluid or hyaluronic acid solutions $(80,83)$ can be used.

Another alternative is tensile loading, where the sample is usually cut to a dumbbell shape, immobilized between two grips and elongated at a constant strain rate while stress is recorded. Typical tensile stress-strain curves of articular cartilage samples show a non-linear "toe region" where the collagen fibers align along the loading direction. Once the fibers are aligned, a linear region follows which corresponds to the stretching of the fibers (5). Tensile Young's modulus, ultimate tensile stress and strain can be obtained from the stress-strain curve (84). In tensile testing of articular cartilage, these parameters are governed by the mechanical properties of the collagen fibers.

Mechanical testing of cartilage has been performed almost exclusively on hyaline and fibrocartilage, and the mechanical parameters obtained are linked to components of the tissues. However, AUC additionally contains an extensive elastic fiber network $(8,85)$. Therefore, it is necessary to consider the functional role of elastin when designing a suitable mechanical evaluation protocol for elastic cartilage.

\section{c. Mechanical testing of elastin-rich tissues}

The functional role of elastin fibers has been mainly investigated in the field of cardiovascular biomechanics, as the aorta and major vascular vessels contain $28-32 \%$ elastin (dry mass)(86). Other tissues rich in elastin include lungs (3-7\% of dry mass), elastic ligaments (50\%), tendons (4\%) and skin (2-3\%) (86). The functional role of elastin is to store elastic-strain energy (87). For example, this allows arteries to smooth the pulsatile flow of blood from the heart, lowering peak blood pressure and maintaining a relatively steady flow of blood through tissues (87). 
In AUC, elastin fibers form a 3D honeycomb network surrounding the tissue chondrocytes $(8,85)$. It can be speculated that the function of this extensive network is to allow large deformations. This is supported by the observations of Roy et al. (88), who characterized the large-deflection bending behavior of porcine cartilage and measured a significantly lower bending modulus in AUC compared to costal cartilage. Further evidence is based on the observation that the size of the human auricle continues to increase with age even after adulthood (89), which may be explained by structural changes in the elastin network (90); specifically elastin degradation. Although a fraction of auricle elongation may be due to lengthening of the earlobe (adipose tissue and skin), a $5 \mathrm{~mm}$ elongation of AUC is observed from subjects in their twenties through to those older than 80 years (91). Ito et al. (90) have presented evidence of several histological changes to cartilage occurring with age. Histological and electron microscopic examination showed that elastic fibers become more heterogeneous in thickness with age. They suggest that these changes are one of the reasons for AUC lengthening. While there are no reports in literature of direct investigation of the functional role of elastin fibers in AUC, Smith et al. (92) have shown in the human annulus fibrosis that enzymatic degradation of elastin fibers significantly reduces initial and ultimate modulus while increasing extensibility, suggesting that elastin fibers functionally important. Additionally, Mansfield et al. (57) have investigated the relationship between the depth-dependent collagen network and the elastin network in articular cartilage using multi-photon microscopy. They demonstrated that elastin fibers are only visible in the superficial zone and the uppermost transitional zone of articular cartilage, where they lie parallel to the predominant collagen fiber orientation. Being largely confined to the surface, which is exposed to high shearing forces, the authors suggest that the elastin network like the collagen network has a mechanical function.

Gosline et al. (87) defined three criteria characterizing proteins similar to elastin (rubber-like proteins): high resilience, large extensibility (strain at failure) and low tensile modulus. Resilience, or elastic efficiency, characterizes how much of the energy required to deform the sample is stored as elastic-strain energy and can be recovered in elastic recoil (87). Extensibility and tensile modulus are measured from tensile stress-strain tests as described above. Resilience can be calculated with a single tensile loading-unloading cycle where it equals the ratio of the area within the stress-strain loop (strain energy dissipated) to the area beneath the loading curve (total strain energy input) (93). Resilience (at $1 \mathrm{~Hz}$ ) of elastin isolated from bovine ligament (ligament nuchae) was measured to be $90 \%$, extensibility $150 \%$ and tensile stiffness $1.1 \mathrm{MPa}(87,94)$. In comparison, collagen fibers $(87,93)$ can hardly be considered as rubber-like proteins since their extensibility is 
only $13 \%$ and their tensile modulus is approximately 1000 times greater than that of elastin (120 MPa), they present however a similar resilience to elastic fibers (90\%). Elastin and collagen have therefore different mechanical properties (95) and the mechanical properties of the tissue will be influenced by the relative composition of elastin and collagen.

However tissue structure, in addition to its composition, determines tissue mechanical properties. Evidence of this is provided, for example, by the relative amount of elastin to collagen in the vascular tree. In dogs, this ratio was shown to be 2 for the thoracic aorta and 0.5 elsewhere $(95,96)$. However, comparison between composition and vessel mechanics is difficult, since it appears that elastin and collagen fibers function in association. It was suggested that elastin determines the stiffness at low extensions, while the collagen forms an inextensible net preventing the vessel from distending beyond the yield point of elastin $(95,96)$. Similarly, recent developments in mathematical modeling of arteries point out that taking both elastin and collagen fiber orientation into account, is needed to understand the mechanical behavior of arteries (97).

Knowledge of the relative orientation of collagen and elastin fibers in AUC could be gained by techniques such as multi-photon microscopy (98), which permits simultaneous imaging of collagen and elastin fibers (99) of, for example, heart vessel leaflet (100), skin (101-103) and articular cartilage samples $(57,104)$. It has the advantage over routine histology in providing 3D information and being non-destructive (99).

In order to establish a benchmark against which to assess the functional competence of engineered AUC constructs, it appears necessary to combine the characterization of the compressive behavior of the tissue matrix with tests capturing the potential contribution of elastic fibers. Therefore the use of routine compressive indentation stress-relaxation tests alongside tensile testing is proposed. Moreover, elastin, collagen, GAG and water content should be quantified to better understand the functional role of each tissue component and the influence of tissue structure. Additionally, the 3D orientation of elastin and collagen fibers should be characterized with - for example - multi-photon microscopy. Past work performed on the mechanical and biochemical characterization of AUC may be helpful in establishing this benchmark.

\section{Auricular cartilage benchmark, past work and recommendations}

Although articular cartilage has been extensively characterized (5), mechanical and biochemical data for AUC are scarce. Mechanical characterization was reported by Naumann et al. (8) who performed immunohistochemical analysis of elastin and collagen types I, II, V,VI and X, biochemical quantification of total GAG content, and mechanical analysis of elastic (auricular and epiglottal), hyaline (nasal and articular) and 
fibrocartilage (meniscus) in New Zealand white rabbits. Creep tests were performed with a plane-ended porous indenter $(\varnothing 0.91 \mathrm{~mm})$ to measure equilibrium modulus, Poisson's ratio and permeability. No significant differences were observed between Poisson's ratio and permeability across the cartilage subtypes suggesting similar behavior in compression. Interestingly, equilibrium modulus did not correlate with cartilage subtype or GAG content. This indicates that tissue or elastin structures, which were not characterized, may be needed to explain these results. Roy et al. (88) performed large deflection three-point bending tests on costal, AUC strips in Yorkshire pigs, and reported significantly lower bending modulus for AUC compared to costal cartilage. Their results also indicate that the perichondrium surrounding AUC may play a functional role. These interesting results support the idea of different mechanics in auricular and hyaline cartilage. The two studies presented above were performed on animal models, and no mechanical data is available comparing human cartilage subtypes. Similarly, several AUC TE studies report mechanical $(31,51)$ or biochemical $(20,31)$ quantification of AUC, yet these measurements were performed on animal material with various mechanical testing protocols and a limited numbers of samples.

Numerous studies present routine histology slices of AUC stained for GAG, collagen and elastin $(4,8,90)$, and report a 3D honeycomb elastin network of elastin fibers extending though the cartilage matrix (8). Yet, no 3D information about fiber orientation has been obtained, while multi-photon microscopy of collagen and elastin fibers was only performed on hyaline cartilage (57).

Little mechanical and biochemical characterization of AUC is available in literature, and no data is available on human AUC. Thus to provide a benchmark for engineered AUC, it would be advantageous to harvest human AUC biopsies, characterize these biopsies with the biochemical and mechanical protocols proposed above, and to work on cohorts large enough to assess the effect of age, gender and sample harvesting location on the measured parameters.

\section{Acknowledgements:}

Thanks to Mieke Pleumeekers at the Department of Otorhinolaryngology, Erasmus MC, University Medical Center Rotterdam, Rotterdam, The Netherlands for preparing ear cartilage histology.

\section{Disclosure Statement:}

No competing financial interests exist. 


\section{References:}

1. Beahm, E.K. and Walton, R.L. Auricular reconstruction for microtia: part I. Anatomy, embryology, and clinical evaluation. Plast Reconstr Surg 109, 2473, 2002.

2. Staffenberg, D.A. Microtia repair. J Craniofac Surg 14, 481, 2003.

3. Ross, M.H., Romrell, L.J. and Kaye, G.I. Histology, A text and atlas. Baltimore: Williams \& Wilkins, 1995, pp. 204.

4. Sayed, K.E., Haisch, A., John, T., Marzahn, U., Lohan, A., Müller, R.D., Kohl, B., Ertel, W., Stoelzel, K. and Schulze-Tanzil, G. Heterotopic autologous chondrocyte transplantation-A realistic approach to support articular cartilage repair? Tissue Eng Part B Rev 16, 603, 2010.

5. Mow, V.C. and Guo, X.E. Mechano-electrochemical properties of articular cartilage: their inhomogeneities and anisotropies. Annu Rev Biomed Eng 4, 175, 2002.

6. Rotter, N., Tobias, G., Lebl, M., Roy, A.K., Hansen, M.C., Vacanti, C.A. and Bonassar, L.J. Age-related changes in the composition and mechanical properties of human nasal cartilage. Arch Biochem Biophys 403 , 132,2002

7. Joshi, M.D., Suh, J.-K., Marui, T. and Woo, S.L.Y. Interspecies variation of compressive biomechanical properties of the meniscus. J Biomed Mater Res 29, 823, 1995.

8. Naumann, A., Dennis, J.E., Awadallah, A., Carrino, D.A., Mansour, J.M., Kastenbauer, E. and Caplan, A.I. Immunochemical and mechanical characterization of cartilage subtypes in rabbit. J Histochem Cytochem 50, 1049, 2002

9. Rotter, N., Steiner, A. and Scheithauer, M. Reconstruction of auricular cartilage using tissueengineering techniques. Operat Tech Otolaryngol Head Neck Surg 19, 278, 2008.

10. Cenzi, R., Farina, A., Zuccarino, L. and Carinci, F. Clinical outcome of 285 Medpor grafts used for craniofacial reconstruction. J Craniofac Surg 16, 526, 2005.

11. Tanzer, R.C. Microtia - a long-term follow-up of 44 reconstructed auricles. Plast Reconstr Surg $\mathbf{6 1 , 1 6 1 ,}$ 1978.

12. Brent, B. Auricular repair with autogenous rib cartilage grafts: two decades of experience with 600 cases. Plast Reconstr Surg 90, 355, 1992.

13. Nagata, S. A new method of total reconstruction of the auricle for microtia. Plast Reconstr Surg 92, 187, 1993. 
14. Firmin, F. Ear reconstruction in cases of typical microtia. Personal experience based on 352 microtic ear corrections. Scand J Plast Reconstr Surg Hand Surg 32, 35, 1998.

15. Romo, T., 3rd, Fozo, M.S. and Sclafani, A.P. Microtia reconstruction using a porous polyethylene framework. Facial Plast Surg 16, 15, 2000.

16. Walton, R.L. and Beahm, E.K. Auricular reconstruction for microtia: part II. surgical techniques. Plast Reconstr Surg 110, 234, 2002.

17. Ohara, K., Nakamura, K. and Ohta, E. Chest wall deformities and thoracic scoliosis after costal cartilage graft harvesting. Plast Reconstr Surg 99, 1030, 1997.

18. Cao, Y., Vacanti, J.P., Paige, K.T., Upton, J. and Vacanti, C.A. Transplantation of chondrocytes utilizing a polymer-cell construct to produce tissue-engineered cartilage in the shape of a human ear. Plast Reconstr Surg 100, 297, 1997.

19. Liu, Y., Zhang, L., Zhou, G., Li, Q., Liu, W., Yu, Z., Luo, X., Jiang, T., Zhang, W. and Cao, Y. In vitro engineering of human ear-shaped cartilage assisted with CAD/CAM technology. Biomaterials 31, 2176, 2010.

20. Shieh, S.-J., Terada, S. and Vacanti, J.P. Tissue engineering auricular reconstruction: in vitro and in vivo studies. Biomaterials 25, 1545, 2004.

21. Cao, Y., Rodriguez, A., Vacanti, M., Ibarra, C., Arevalo, C. and Vacanti, C.A. Comparative study of the use of poly(glycolic acid), calcium alginate and pluronics in the engineering of autologous porcine cartilage. J Biomater Sci Polym Ed 9, 475, 1998.

22. Kamil, S.H., Vacanti, M.P., Aminuddin, B.S., Jackson, M.J., Vacanti, C.A. and Eavey, R.D. Tissue engineering of a human sized and shaped auricle using a mold. Laryngoscope 114, 867, 2004.

23. Haisch, A., Klaring, S., Groger, A., Gebert, C. and Sittinger, M. A tissue-engineering model for the manufacture of auricular-shaped cartilage implants. Eur Arch Otorhinolaryngol 259, 316, 2002.

24. Isogai, N., Morotomi, T., Hayakawa, S., Munakata, H., Tabata, Y., Ikada, Y. and Kamiishi, H. Combined chondrocyte-copolymer implantation with slow release of basic fibroblast growth factor for tissue engineering an auricular cartilage construct. J Biomed Mater Res A 74, 408, 2005.

25. Kusuhara, H., Isogai, N., Enjo, M., Otani, H., Ikada, Y., Jacquet, R., Lowder, E. and Landis, W.J. Tissue engineering a model for the human ear: assessment of size, shape, morphology, and gene expression following seeding of different chondrocytes. Wound Repair Regen 17, 136, 2009. 
26. Isogai, N., Asamura, S., Higashi, T., Ikada, Y., Morita, S., Hillyer, J., Jacquet, R. and Landis, W.J. Tissue engineering of an auricular cartilage model utilizing cultured chondrocyte-poly(L-lactide-epsilon-caprolactone) scaffolds. Tissue Eng 10, 673, 2004.

27. Isogai, N., Nakagawa, Y., Suzuki, K., Yamada, R., Asamura, S., Hayakawa, S. and Munakata, H. Cytokinerich autologous serum system for cartilaginous tissue engineering. Ann Plast Surg 60, 703, 2008.

28. Saim, A.B., Cao, Y., Weng, Y., Chang, C.N., Vacanti, M.A., Vacanti, C.A. and Eavey, R.D. Engineering autogenous cartilage in the shape of a helix using an injectable hydrogel scaffold. Laryngoscope 110, 1694, 2000.

29. Xu, J.W., Johnson, T.S., Motarjem, P.M., Peretti, G.M., Randolph, M.A. and Yaremchuk, M.J. Tissueengineered flexible ear-shaped cartilage. Plast Reconstr Surg 115, 1633, 2005.

30. Ting, V., Sims, C.D., Brecht, L.E., McCarthy, J.G., Kasabian, A.K., Connelly, P.R., Elisseeff, J., Gittes, G.K. and Longaker, M.T. In vitro prefabrication of human cartilage shapes using fibrin glue and human chondrocytes. Ann Plast Surg 40, 413, 1998.

31. Chang, S.C., Tobias, G., Roy, A.K., Vacanti, C.A. and Bonassar, L.J. Tissue engineering of autologous cartilage for craniofacial reconstruction by injection molding. Plast Reconstr Surg 112, 793, 2003.

32. Neumeister, M.W., Wu, T. and Chambers, C. Vascularized tissue-engineered ears. Plast Reconstr Surg 117, 116, 2006.

33. Zhou, L., Pomerantseva, I., Bassett, E.K., Bowley, C.M., Zhao, X., Bichara, D.A., Kulig, K.M., Vacanti, J.P., Randolph, M.A. and Sundback, C.A. Engineering ear constructs with a composite scaffold to maintain dimensions. Tissue Eng Part A 17, 1573, 2011.

34. Lee, S.J., Broda, C., Atala, A. and Yoo, J.J. Engineered cartilage covered ear implants for auricular cartilage reconstruction. Biomacromolecules 12, 306, 2011.

35. Ruszymah, B.H., Chua, K.H., Mazlyzam, A.L. and Aminuddin, B.S. Formation of tissue engineered composite construct of cartilage and skin using high density polyethylene as inner scaffold in the shape of human helix. Int J Pediatr Otorhinolaryngol 75, 805, 2011.

36. Bichara, D.A., Zhao, X., Hwang, N.S., Bodugoz-Senturk, H., Yaremchuk, M.J., Randolph, M.A. and Muratoglu, O.K. Porous poly(vinyl alcohol)-alginate gel hybrid construct for neocartilage formation using human nasoseptal cells. J Surg Res 163, 331, 2010. 
37. Yanaga, H., Imai, K., Fujimoto, T. and Yanaga, K. Generating ears from cultured autologous auricular chondrocytes by using two-stage implantation in treatment of microtia. Plast Reconstr Surg 124, 817, 2009.

38. Bichara, D.A., O'Sullivan, N.A., Pomerantseva, I., Zhao, X., Sundback, C.A., Vacanti, J. and Randolph, M.A. The tissue engineered auricle: past, present and future. Tissue Eng Part B Rev, 2011.

39. Abbott, J. and Holtzer, H. The loss of phenotypic traits by differentiated cells. 3 . The reversible behavior of chondrocytes in primary cultures. J Cell Biol 28, 473, 1966.

40. Holtzer, H., Abbott, J., Lash, J. and Holtzer, S. The loss of phenotypic traits by differentiated cells in vitro, I. Dedifferentiation of cartilage cells. Proc Natl Acad Sci 46, 1533, 1960.

41. Homicz, M.R., Schumacher, B.L., Sah, R.L. and Watson, D. Effects of serial expansion of septal chondrocytes on tissue-engineered neocartilage composition. Otolaryngol Head Neck Surg 127, $398,2002$.

42. Mandl, E.W., van der Veen, S.W., Verhaar, J.A. and van Osch, G.J. Multiplication of human chondrocytes with low seeding densities accelerates cell yield without losing redifferentiation capacity. Tissue Eng 10, 109, 2004.

43. van Osch, G.J., van der Veen, S.W. and Verwoerd-Verhoef, H.L. In vitro redifferentiation of cultureexpanded rabbit and human auricular chondrocytes for cartilage reconstruction. Plast Reconstr Surg 107, 433, 2001.

44. van Osch, G.J., Mandl, E.W., Jahr, H., Koevoet, W., Nolst-Trenite, G. and Verhaar, J.A. Considerations on the use of ear chondrocytes as donor chondrocytes for cartilage tissue engineering. Biorheology $\mathbf{4 1 , 4 1 1 ,}$ 2004.

45. Mandl, E.W., van der Veen, S.W., Verhaar, J.A. and van Osch, G.J. Serum-free medium supplemented with high-concentration FGF2 for cell expansion culture of human ear chondrocytes promotes redifferentiation capacity. Tissue Eng 8, 573, 2002.

46. Mandl, E.W., Jahr, H., Koevoet, J.L., van Leeuwen, J.P., Weinans, H., Verhaar, J.A. and van Osch, G.J. Fibroblast growth factor-2 in serum-free medium is a potent mitogen and reduces dedifferentiation of human ear chondrocytes in monolayer culture. Matrix Biol 23, 231, 2004.

47. Guilak, F., Awad, H.A., Fermor, B., Leddy, H.A. and Gimble, J.M. Adipose-derived adult stem cells for cartilage tissue engineering. Biorheology 41, 389, 2004.

48. Song, L., Baksh, D. and Tuan, R.S. Mesenchymal stem cell-based cartilage tissue engineering: cells, scaffold and biology. Cytotherapy 6, 596, 2004 
49. Kamil, S.H., Woda, M., Bonassar, L.J., Novitsky, Y.W., Vacanti, C.A., Eavey, R.D. and Vacanti, M.P. Normal features of tissue-engineered auricular cartilage by flow cytometry and histology: patient safety. Otolaryngol Head Neck Surg 129, 390, 2003.

50. Arevalo-Silva, C.A., Eavey, R.D., Cao, Y., Vacanti, M., Weng, Y. and Vacanti, C.A. Internal support of tissue-engineered cartilage. Arch Otolaryngol Head Neck Surg 126, 1448, 2000.

51. Britt, J.C. and Park, S.S. Autogenous tissue-engineered cartilage: evaluation as an implant material. Arch Otolaryngol Head Neck Surg 124, 671, 1998.

52. Mow, V.C., Ratcliffe, A. and Poole, A.R. Cartilage and diarthrodial joints as paradigms for hierarchical materials and structures. Biomaterials 13, 67, 1992.

53. Cotta-Pereira, G., Del-Caro, L.M. and Montes, G.S. Distribution of elastic system fibers in hyaline and fibrous cartilages of the rat. Acta Anat (Basel) 119, 80, 1984.

54. Yu, J., Winlove, P.C., Roberts, S. and Urban, J.P. Elastic fibre organization in the intervertebral discs of the bovine tail. J Anat 201, 465, 2002.

55. Yu, J., Tirlapur, U., Fairbank, J., Handford, P., Roberts, S., Winlove, C.P., Cui, Z. and Urban, J. Microfibrils, elastin fibres and collagen fibres in the human intervertebral disc and bovine tail disc. J Anat 210, 460, 2007.

56. Yeh, A.T., Hammer-Wilson, M.J., Van Sickle, D.C., Benton, H.P., Zoumi, A., Tromberg, B.J. and Peavy, G.M. Nonlinear optical microscopy of articular cartilage. Osteoarthritis Cartilage 13, 345, 2005.

57. Mansfield, J., Yu, J., Attenburrow, D., Moger, J., Tirlapur, U., Urban, J., Cui, Z. and Winlove, P. The elastin network: its relationship with collagen and cells in articular cartilage as visualized by multiphoton microscopy. J Anat 215, 682, 2009.

58. Yu, J. and Urban, J.P. The elastic network of articular cartilage: an immunohistochemical study of elastin fibres and microfibrils. J Anat 216, 533, 2010.

59. Ross, R. The elastic fiber a review. Journal of Histochemistry \& Cytochemistry 21, 199, 1973.

60. Vrhovski, B. and Weiss, A.S. Biochemistry of tropoelastin. Eur J Biochem 258, 1, 1998.

61. Montes, G.S. Structural biology of the fibres of the collagenous and elastic systems. Cell Biol Int 20, 15, 1996.

62. Gibson, M.A., Kumaratilake, J.S. and Cleary, E.G. The protein components of the 12-nanometer microfibrils of elastic and nonelastic tissues. J Biol Chem 264, 4590, 1989. 
63. Sakai, L.Y., Keene, D.R. and Engvall, E. Fibrillin, a new 350-kD glycoprotein, is a component of extracellular microfibrils. J Cell Biol 103, 2499, 1986.

64. Ross, R. and Bornstein, P. The elastic fiber. I. The separation and partial characterization of its macromolecular components. J Cell Biol 40, 366, 1969.

65. Cotta-Pereira, G., Rodrigo, G. and Bittencourt-Sampaio, S. Oxytalan, elaunin, and elastic fibers in the human skin. J Investig Dermatol 66, 143, 1976.

66. Sherratt, M.J., Baldock, C., Louise Haston, J., Holmes, D.F., Jones, C.J.P., Adrian Shuttleworth, C., Wess, T.J. and Kielty, C.M. Fibrillin microfibrils are stiff reinforcing fibres in compliant tissues. J Mol Biol 332, 183, 2003.

67. Bartel DL, Davy DT and Keaveny TM. Orthopaedic biomechanics: mechanics and design in musculoskeletal systems. Upper Saddle River, NJ: Pearson Prentice Hall, 2006, pp. 121.

68. Eyre, D.R. and Muir, H. Types I and II collagens in intervertebral disc. Interchanging radial distributions in annulus fibrosus. Biochem J 157, 267, 1976.

69. Eyre, D.R. Collagen of articular cartilage. Arthritis Res 4, 30, 2002.

70. Eyre, D.R. and Muir, H. The distribution of different molecular species of collagen in fibrous, elastic and hyaline cartilages of the pig. Biochem J 151, 595, 1975.

71. Bastiaansen-Jenniskens, Y.M., Koevoet, W., de Bart, A.C., van der Linden, J.C., Zuurmond, A.M., Weinans, H., Verhaar, J.A., van Osch, G.J. and Degroot, J. Contribution of collagen network features to functional properties of engineered cartilage. Osteoarthr Cartil 16, 359, 2008.

72. Elbjeirami, W.M., Yonter, E.O., Starcher, B.C. and West, J.L. Enhancing mechanical properties of tissueengineered constructs via lysyl oxidase crosslinking activity. J Biomed Mater Res A 66, 513, 2003.

73. Armstrong, C.G. An analysis of the stresses in a thin layer of articular cartilage in a synovial joint. Eng Med 15, 55, 1986.

74. Armstrong, C.G. and Mow, V.C. Variations in the intrinsic mechanical properties of human articular cartilage with age, degeneration, and water content. J Bone Joint Surg Am 64, 88, 1982.

75. Little, C.J., Bawolin, N.K. and Chen, X.B. Mechanical properties of natural cartilage and tissueengineered constructs. Tissue Eng Part B Rev 17, 213, 2011. 
76. Buschmann, M.D., Soulhat, J., Shirazi-Adl, A., Jurvelin, J.S. and Hunziker, E.B. Confined compression of articular cartilage: linearity in ramp and sinusoidal tests and the importance of interdigitation and incomplete confinement. J Biomech 31, 171, 1998.

77. DiSilvestro, M.R. and Suh, J.K. A cross-validation of the biphasic poroviscoelastic model of articular cartilage in unconfined compression, indentation, and confined compression. J Biomech 34, 519, 2001.

78. Hayes, W.C., Keer, L.M., Herrmann, G. and Mockros, L.F. A mathematical analysis for indentation tests of articular cartilage. J Biomech 5, 541, 1972.

79. Mow, V.C., Gibbs, M.C., Lai, W.M., Zhu, W.B. and Athanasiou, K.A. Biphasic indentation of articular cartilage--II. A numerical algorithm and an experimental study. J Biomech 22, 853, 1989.

80. Korhonen, R.K., Laasanen, M.S., Toyras, J., Rieppo, J., Hirvonen, J., Helminen, H.J. and Jurvelin, J.S. Comparison of the equilibrium response of articular cartilage in unconfined compression, confined compression and indentation. J Biomech 35, 903, 2002.

81. Stok, K.S., Lisignoli, G., Cristino, S., Facchini, A. and Muller, R. Mechano-functional assessment of human mesenchymal stem cells grown in three-dimensional hyaluronan-based scaffolds for cartilage tissue engineering. J Biomed Mater Res A 93, 37, 2010.

82. Spilker, R.L., Suh, J.K. and Mow, V.C. A finite element analysis of the indentation stress-relaxation response of linear biphasic articular cartilage. J Biomech Eng 114, 191, 1992.

83. Armstrong, C.G., Lai, W.M. and Mow, V.C. An analysis of the unconfined compression of articular cartilage. J Biomech Eng 106, 165, 1984.

84. Williamson, A.K., Chen, A.C., Masuda, K., Thonar, E.J. and Sah, R.L. Tensile mechanical properties of bovine articular cartilage: variations with growth and relationships to collagen network components. J Orthop Res 21, 872, 2003

85. Bradamante, Z., Kostovic-Knezevic, L., Levak-Svajger, B. and Svajger, A. Differentiation of the secondary elastic cartilage in the external ear of the rat. Int J Dev Biol 35, 311, 1991.

86. Uitto, J. Biochemistry of the elastic fibers in normal connective tissues and its alterations in diseases. J Invest Dermatol 72, 1, 1979.

87. Gosline, J., Lillie, M., Carrington, E., Guerette, P., Ortlepp, C. and Savage, K. Elastic proteins: biological roles and mechanical properties. Philos Trans R Soc Lond B Biol Sci 357, 121, 2002. 
88. Roy, R., Kohles, S.S., Zaporojan, V., Peretti, G.M., Randolph, M.A., Xu, J. and Bonassar, L.J. Analysis of bending behavior of native and engineered auricular and costal cartilage. J Biomed Mater Res A 68, 597, 2004. 89. Pellnitz, D. Über das Wachstum der menschlichen Ohrmuschel. Eur Arch Otorhinolaryngol 171, 334, 1958.

90. Ito, I., Imada, M., Ikeda, M., Sueno, K., Arikuni, T. and Kida, A. A morphological study of age changes in adult human auricular cartilage with special emphasis on elastic fibers. Laryngoscope 111, 881, 2001.

91. Meijerman, L., van der Lugt, C. and Maat, G.J. Cross-sectional anthropometric study of the external ear. J Forensic Sci 52, 286, 2007.

92. Smith, L.J., Byers, S., Costi, J.J. and Fazzalari, N.L. Elastic fibers enhance the mechanical integrity of the human lumbar anulus fibrosus in the radial direction. Ann Biomed Eng 36, 214, 2008.

93. Pollock, C.M. and Shadwick, R.E. Relationship between body mass and biomechanical properties of limb tendons in adult mammals. Am J Physiol 266, R1016, 1994.

94. Aaron, B.B. and Gosline, J.M. Elastin as a random-network elastomer: A mechanical and optical analysis of single elastin fibers. Biopolymers 20, 1247, 1981

95. Fung, Y.C. Biomechanics : mechanical properties of living tissues. New York: Springer-Verlag, 1993, pp. 196.

96. Harkness, M.L., Harkness, R.D. and McDonald, D.A. The collagen and elastin content of the arterial wall in the dog. Proc R Soc Lond B Biol Sci 146, 541, 1957.

97. Holzapfel, G.A. and Ogden, R.W. Constitutive modelling of arteries. Proc Math Phys Eng Sci 466, 1551, 2010.

98. Helmchen, F. and Denk, W. Deep tissue two-photon microscopy. Nat Methods 2, 932, 2005.

99. Schenke-Layland, K., Riemann, I., Damour, O., Stock, U.A. and Konig, K. Two-photon microscopes and in vivo multiphoton tomographs--powerful diagnostic tools for tissue engineering and drug delivery. Adv Drug Deliv Rev 58, 878, 2006.

100. Schenke-Layland, K., Riemann, I., Stock, U.A. and Konig, K. Imaging of cardiovascular structures using near-infrared femtosecond multiphoton laser scanning microscopy. J Biomed Opt 10, 024017, 2005.

101. Zhuo, S., Chen, J., Jiang, X., Cheng, X. and Xie, S. Visualizing extracellular matrix and sensing fibroblasts metabolism in human dermis by nonlinear spectral imaging. Skin Res Technol 13, 406, 2007. 
102. Koehler, M.J., Hahn, S., Preller, A., Elsner, P., Ziemer, M., Bauer, A., Konig, K., Buckle, R., Fluhr, J.W. and Kaatz, M. Morphological skin ageing criteria by multiphoton laser scanning tomography: non-invasive in vivo scoring of the dermal fibre network. Exp Dermatol 17, 519, 2008.

103. Hanson, K.M. and Bardeen, C.J. Application of nonlinear optical microscopy for imaging skin. Photochem Photobiol 85, 33, 2009.

104. Brockbank, K.G., MacLellan, W.R., Xie, J., Hamm-Alvarez, S.F., Chen, Z.Z. and Schenke-Layland, K. Quantitative second harmonic generation imaging of cartilage damage. Cell Tissue Bank 9, 299, 2008.

105. Bank, R.A., Groener, J.E., van Gemund, J.J., Maaswinkel, P.D., Hoeben, K.A., Schut, H.A. and Everts, V. Deficiency in $\mathrm{N}$-acetylgalactosamine-6-sulfate sulfatase results in collagen perturbations in cartilage of Morquio syndrome A patients. Mol Genet Metab 97, 196, 2009. 


\section{Figure captions:}

Figure 1: Anatomy of the elastic cartilage of the ear: (a) anterior; helicis (H), superior crus (SC), triangular fossa (TF), antihelix (A), cymba conchae (CY), cauda helicis (CU), cavum conchae (C), lamina tragi (LT). (b) posterior; transverse sulcus (TS), ponticulus (P). The auricular muscles: (c-d) the three extrinsic muscles - anterior (A), superior (S), and posterior (P); and the six intrinsic muscles - large (HL) and small (HS) helical muscles, muscle of the tragus (T), muscle of the antitragus (AT), transverse muscle (TM), oblique muscle (O). Adapted from Beahm, E.K., Walton, R.L., 2002. Auricular reconstruction for microtia: part I. Anatomy, embryology, and clinical evaluation. Plast Reconstr Surg 109, 2473-2482 with permission of the publisher.

Figure 2: Schematic overview of the distribution and types of cartilage found in the human body. Adapted from Sayed, K.E., Haisch, A., John, T., Marzahn, U., Lohan, A., Müller, R.D., Kohl, B., Ertel, W., Stoelzel, K., SchulzeTanzil, G., 2010. Heterotopic autologous chondrocyte transplantation-A realistic approach to support articular cartilage repair? Tissue Eng Part B Rev 16, 603-616 with permission of the publisher.

Figure 3: Sketch of the construction of an autologous cartilage graft (i.e. costal cartilage) used for surgical ear reconstruction. Four pieces are removed and carved from the ribs (1-4), then reconstructed to form the outer ear form. Adapted from Pan, B., Jiang, H., Guo, D., Huang, C., Hu, S., Zhuang, H., 2008. Microtia: ear reconstruction using tissue expander and autogenous costal cartilage. J Plast Reconstr Aesthet Surg 61, S98S103 with permission of the publisher.

Figure 4: (a) Approximate schematic of the three phases of elastic cartilage: the porous ECM; including proteoglycans aggregates, elastic fibers, collagen fibers; the water, and the charged ions. These three phases work together to produce an osmotic swelling pressure which allows the cartilage to withstand compressive loading. (b) The molecular structure of the proteoglycan aggregate; which carries charged sulfate groups on its GAG side chains responsible for maintaining the osmotic swelling pressure of the tissue. (c) The molecular structure of the elastic fiber; the core of the fiber is composed of elastin, which is suspected to play a role in tissue resilience and whereas the microfibrils surrounding the elastin core are believed to anchor the fiber in the surrounding matrix. (d) The molecular structure of the collagen fiber (d-period regular stripes visible by electron microscopy are represented); collagen fibers govern the tensile mechanical properties of cartilage. 
Figure 5: Histology of the elastic cartilage components: (a) overview slice (H\&E stain) showing the high cell density of elastic cartilage, (b) Safranin O stain for GAG showing an homogeneous distribution of GAG through the tissue matrix, (c) Resorcin Fuchsin stain for elastic fibers; inset: the elastic fibers form a honeycomb network (white arrows) surrounding the tissue chondrocytes, and (d) immunohistochemistry for collagen II; collagen II is the predominant collagen type in elastic cartilage and is homogeneously distributed through the tissue.

Figure 6: Sketch of the cross-section of an (a) oxytalan fiber, (b) elaunin fiber, (c) elastic fiber and collagen fiber (d). Oxytalan fibers are devoid of elastin. In elastic and elaunin fibers, the elastin core is surrounded by microfibrils. Elastin is more abundant and homogeneous in the elastic fiber than in the elaunin fiber. The sketches are based on electron micrographs $(65,105)$. 


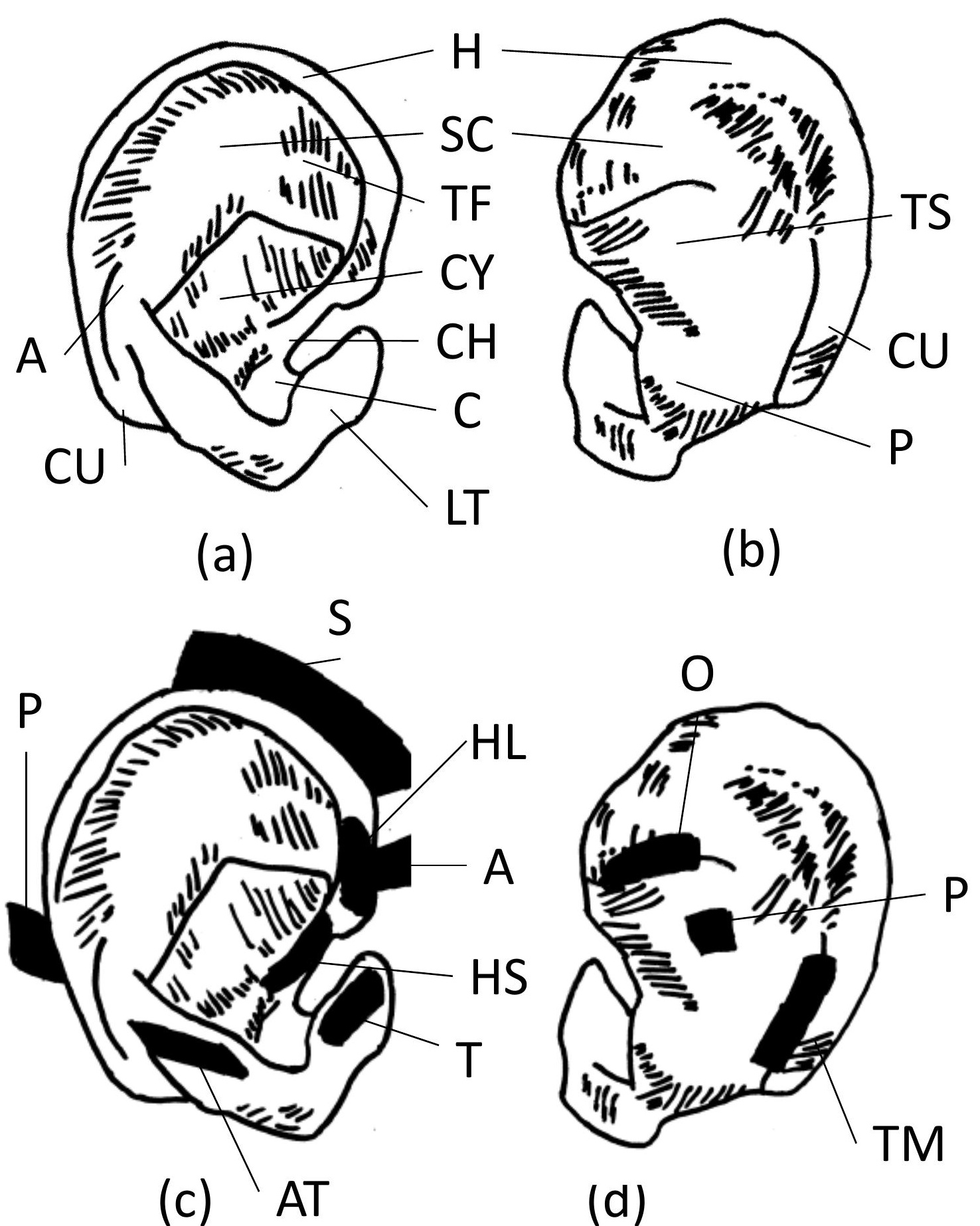

Figure 1 


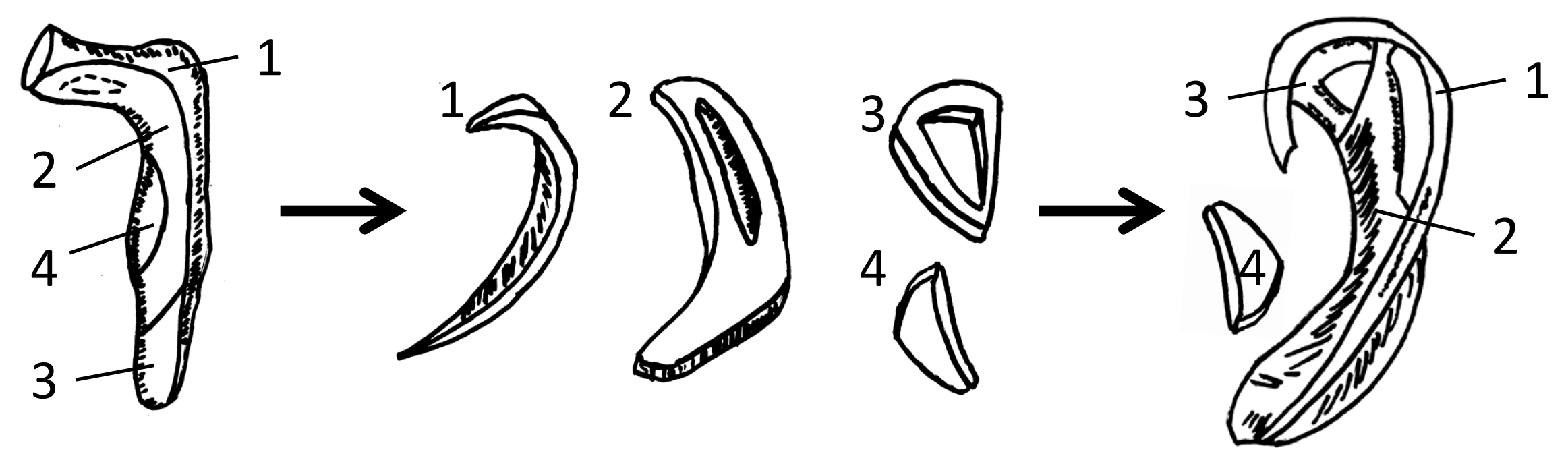

Figure 3 
(a) Elastic cartilage matrix

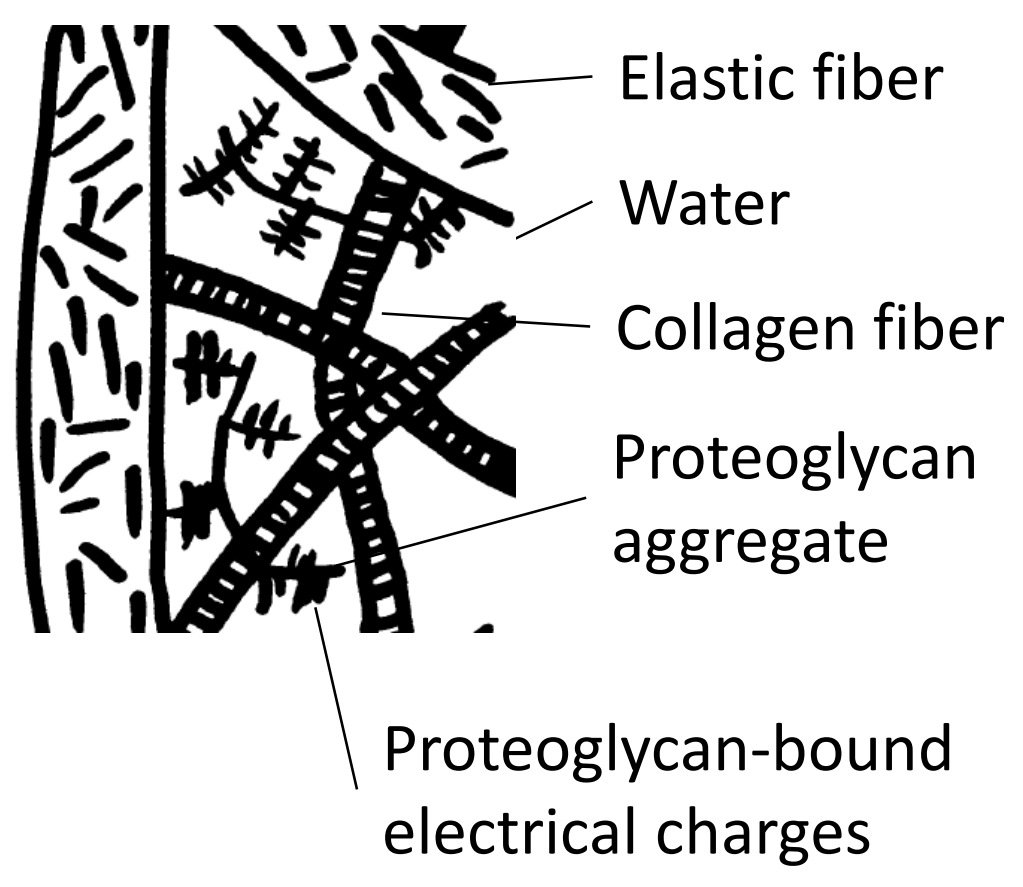

(c) Elastic fiber

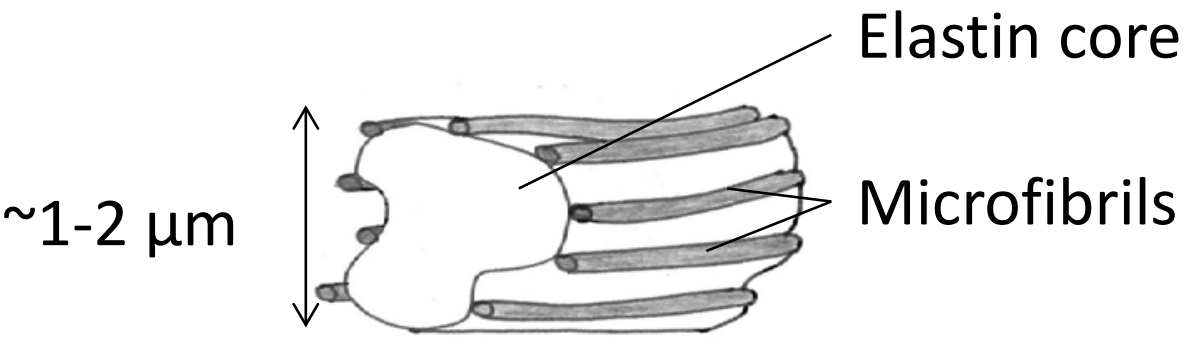

(b) Proteoglycan aggregate

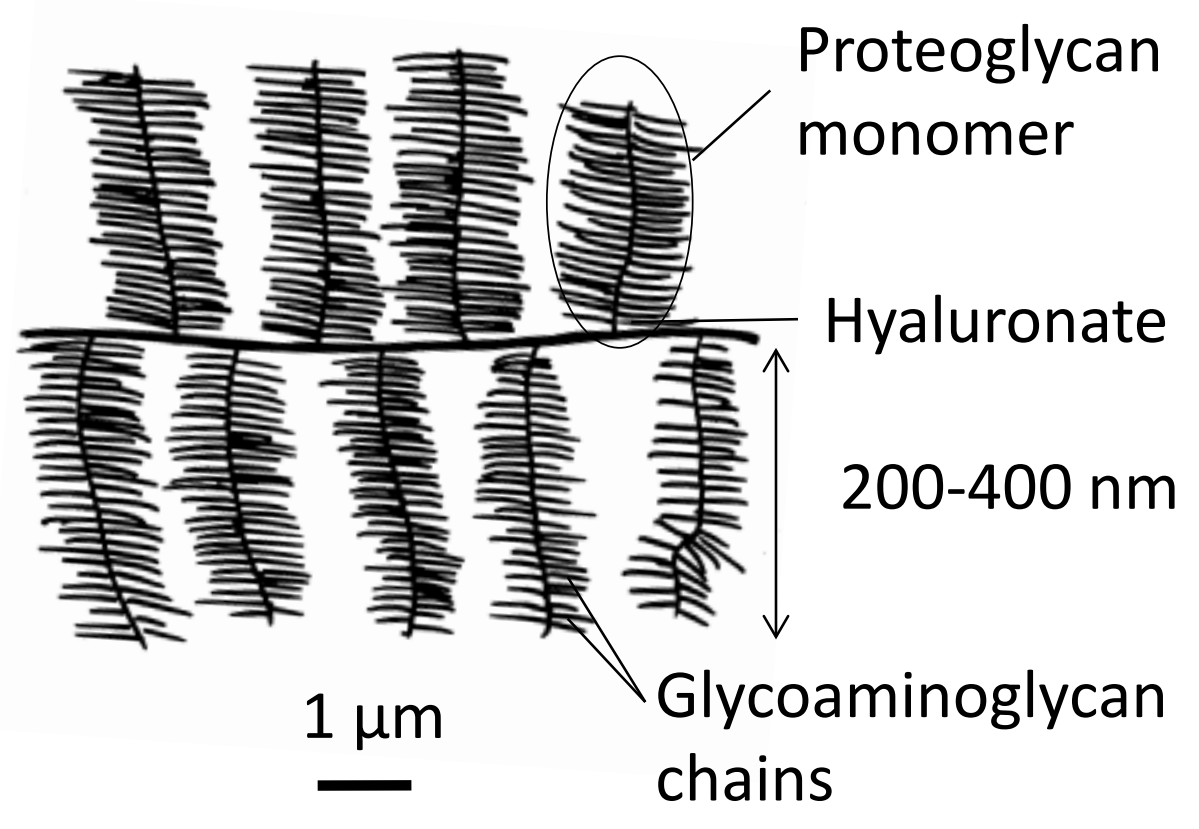

(d) Collagen fiber

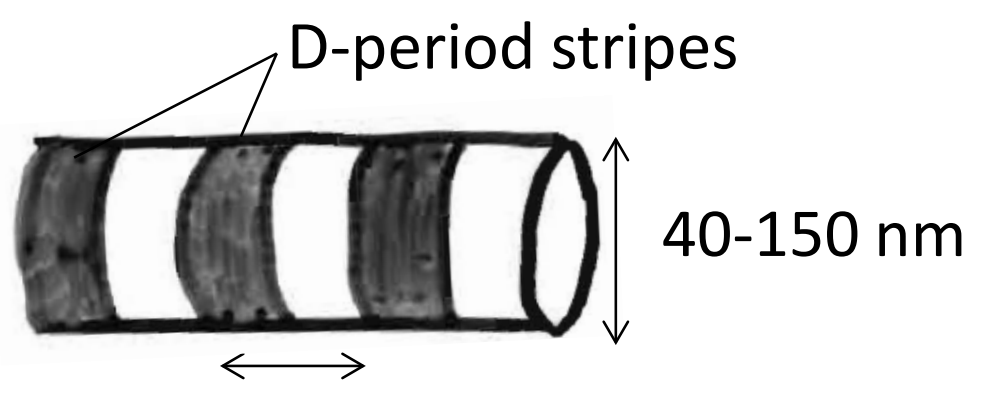

$67 \mathrm{~nm}$

Figure 4 
(a)

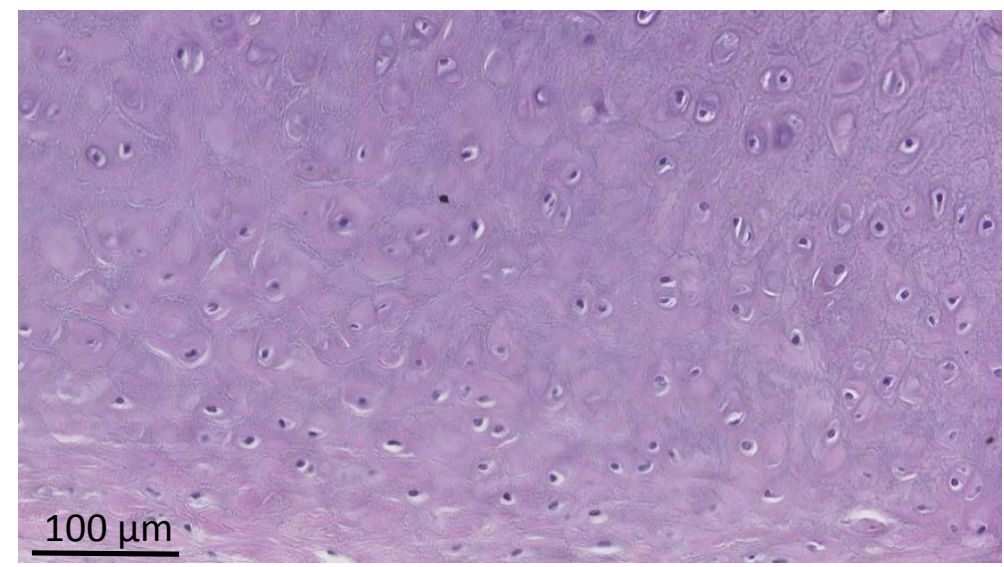

(c)

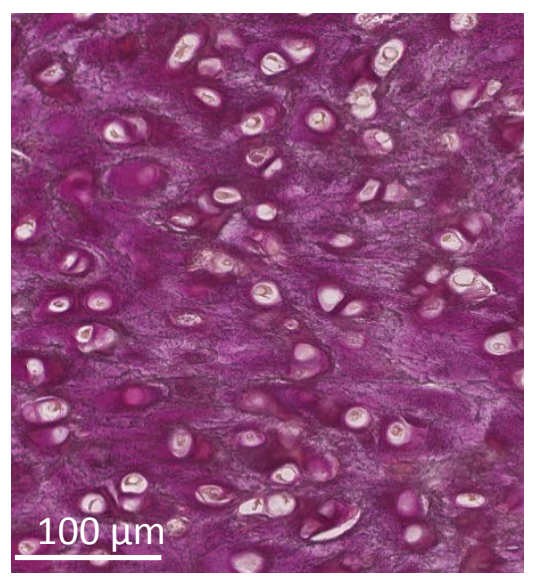

(b)

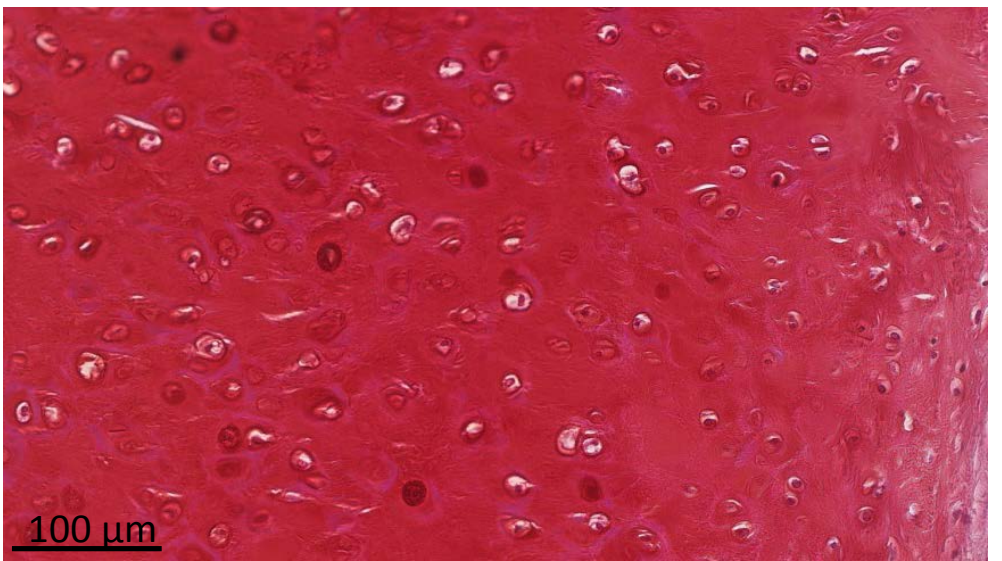

(d)

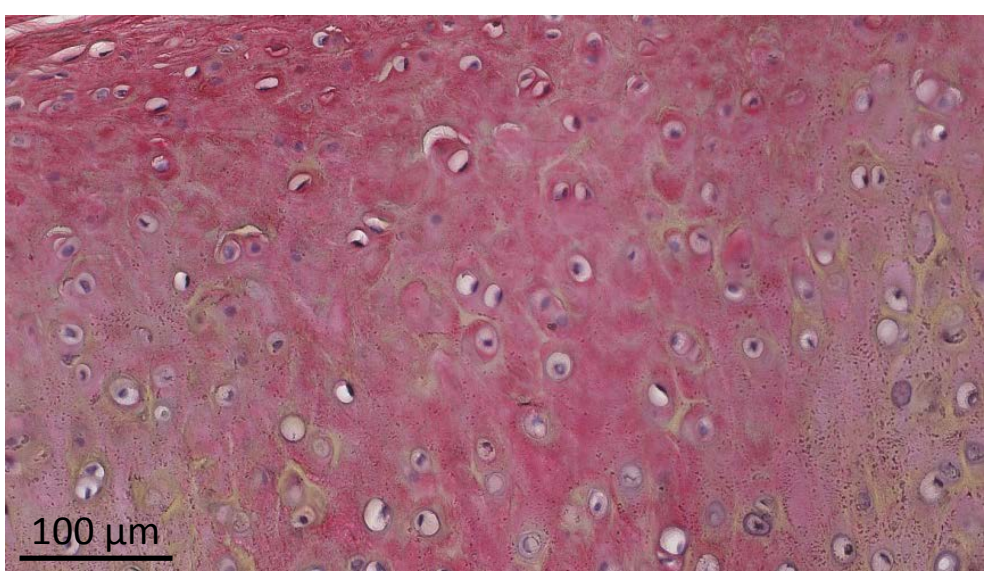

Figure 5 
(a) Oxytalan fiber

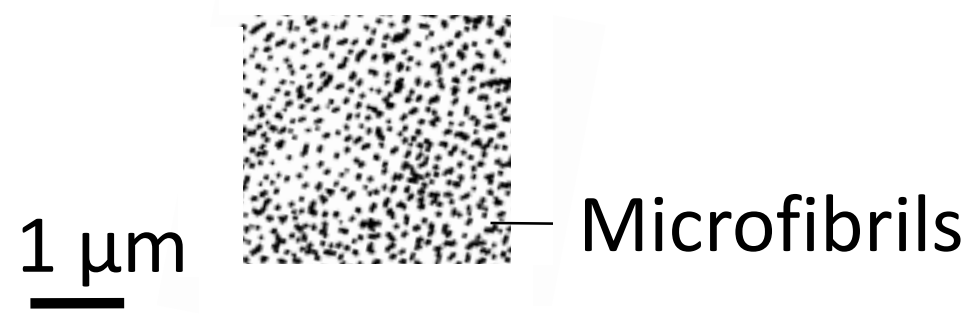

(c) Elastic fiber

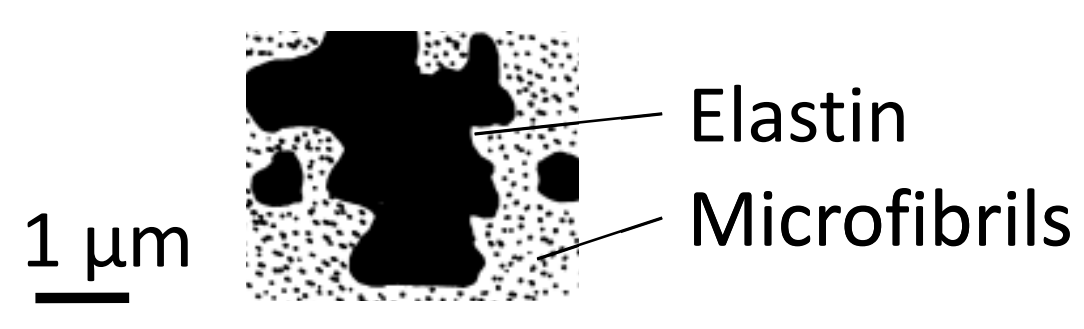

(b) Elaunin fiber

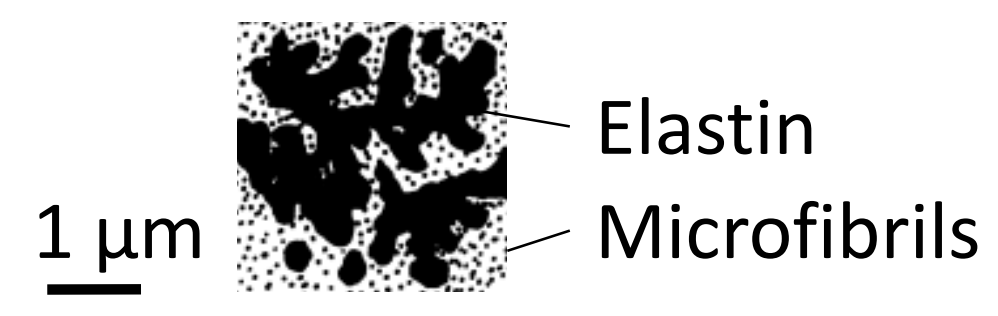

(d) Collagen fibers

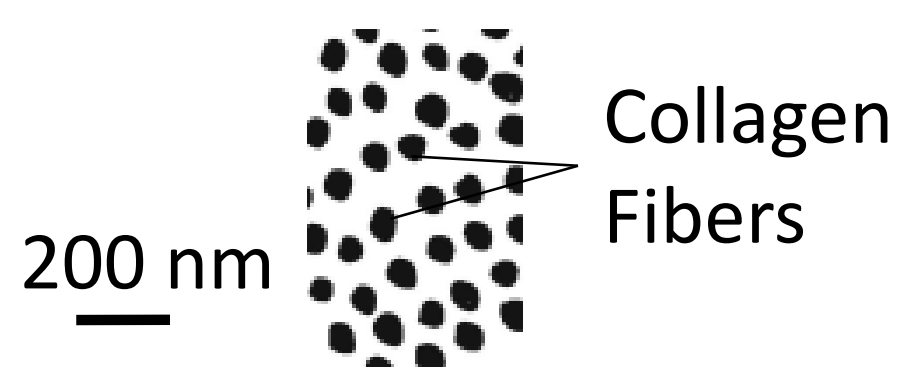

